\title{
Covid-19: Doctors face shortages of vital drugs, gases, and therapeutics, survey finds
}

\author{
Abi Rimmer
}

The BMJ

Shortages of items such as antibiotics, anaesthetic drugs, and painkillers have forced doctors to offer their patients less effective treatments, a BMA survey has found.

The survey, which received 16343 responses from doctors across the UK, was conducted between 28 and 30 April.

Of the 14028 doctors who answered a question on shortages of medicines, medical gases, and other therapeutics, 4\% (579) said they experienced such shortages "most of the time." A further $24 \%$ (3362) said they experienced shortages "some of the time."

A third (2671 of 8373) of doctors said they faced shortages of inhalers, while $27 \%$ (2283) reported shortages of hormone replacement therapy drugs, and 15\% (1266) reported shortages of opioids.

When asked whether these shortages were forcing them to provide less effective treatment to their patients, 5\% (708 of 13 764) said that they frequently did, while $26 \%$ (3620) said that they occasionally did.

The survey also asked doctors about the impact that covid-19 was having on other services.

A third (4155 of 13 846) of respondents said that prioritisation of patients with possible or confirmed covid-19 had significantly worsened the care available to patients with no covid-19 symptoms. A further quarter $(26 \%, 3626)$ said that it had slightly worsened their care.
Chaand Nagpaul, BMA council chair, said the findings bore out the fears held by many doctors that parts of the health service were being neglected.

"This means many patients are not getting the care they need now and, crucially, are risking their conditions getting worse, with some maybe dying as a result," Nagpaul said.

"Once this current situation eases, it's likely that there will be a sudden spike in demand from patients with far more acute illnesses caused by a delay in timely treatment. It's vital that the NHS plans for this now."

He called for a clear plan of how the health system would deal with the ongoing demands of the pandemic, as well as resuming services for those whose care has effectively been frozen.

An NHS spokesperson said, "To some extent this BMA opinion poll is a sad statement of the obvious-like every other health service around the world, hospitals in this country have had to adapt quickly to deal with the greatest public health threat in over a century.

"It's a source of pride for the NHS that every coronavirus patient who needs hospital care has been able to get it. And NHS emergency services have been available to all patients-with or without covid-19-throughout this pandemic. Now, as the number of people needing coronavirus care in hospitals is beginning to fall, other non-urgent services can turn back on." 